\title{
Cryptosporidium and other intestinal parasitic infections among HIV patients in southern Ethiopia: significance of improved HIV-related care
}

\author{
Techalew Shimelis", Yayehyirad Tassachew and Tariku Lambiyo
}

\begin{abstract}
Background: Intestinal parasitic infections are known to cause gastroenteritis, leading to higher morbidity and mortality, particularly in people living with HIV/AIDS. This study aimed to determine the prevalence of Cryptosporidium and other intestinal parasitic infections among HIV patients receiving care at a hospital in Ethiopia where previous available baseline data helps assess if improved HIV-related care has reduced infection rates.

Methods: A cross-sectional study was conducted at Hawassa University Hospital in southern Ethiopia from May, 2013 to March, 2014. A consecutive sample of 491 HIV- infected patients with diarrhea or a CD4 T cell count $<200$ cells/ $\mu$ l were prospectively studied. A single stool sample was collected from each study participant and processed using direct, formolether concentration, and modified Ziehl-Neelsen techniques for the diagnosis of Cryptosporidium and other intestinal parasites. The study was approved by the Institutional Review Board of the College of Medicine and Health Sciences, Hawassa University. Physicians managed participants found to be infected with any pathogenic intestinal parasite.

Results: The overall prevalence of intestinal parasitic infections among the study population was $35.8 \%$. The most prevalent parasites were Cryptosporidium (13.2 \%), followed by Entamoeba histolytica/dispar (10.2\%), and Giardia lamblia (7.9\%). The rate of single and multiple infections were 25.5 and $10.3 \%$, respectively. Patients with a CD4 T cell count $<200$ cells $/ \mu$ l had a similar rate of any intestinal parasitic infection or cryptosporidiosis compared to those with counts $\geq 200$ cells/ $\mu$ l, but with some type of diarrhea.

Conclusion: The study shows high prevalence of intestinal parasitic infections in the study population. However, the results in the current report are significantly lower compared to previous findings in the same hospital. The observed lower infection rate is encouraging and supports the need to strengthen and sustain the existing intervention measures in order to further reduce intestinal parasitic infections in people living with HIV/AIDS.
\end{abstract}

Keywords: Cryptosporidium, Intestinal parasites, HIV/AIDS, Ethiopia

\section{Background}

Cryptosporidium and other parasitic infections are known to cause gastroenteritis and malnutrition, which ultimately leads to significant morbidity and mortality, particularly in people living with human immunodeficiency virus/acquired immunodeficiency syndrome (HIV/ AIDS). Studies have shown that about $30-60 \%$ of AIDS patients in developed countries and $90 \%$ in developing

* Correspondence: techalew03@yahoo.com

Department of Medical Laboratory Science, Hawassa University, P. O. Box 1560, Hawassa, Ethiopia countries experience diarrhea [1]. Bacteria, parasites, fungi and viruses are known etiologic agents of diarrhea, although those of parasitic origin are most prominent in patients with AIDS in developing countries [2].

A group of parasites that came into prominence in humans with the emergence of HIV infection are among known pathogens that cause diarrhea [3]. In association with progressive decline in immunological responses in HIV-infected patients, the epidemiology of these parasites has been intensified. Previous studies showed that HIV patients, particularly those with a lower CD4 T cell 
count, have higher prevalence of opportunistic intestinal parasites such as Cryptosporidium species, Isospora belli, Cyclospora cayetanensis, microsporidia, and Strongyloides stercoralis $[4,5]$.

Cryptosporidium is a ubiquitous coccidian parasite infecting humans and a wide range of domestic and wild animals [6]. The parasite is robust to various chemicals and environmental stresses, lacks host-specificity, has a direct life-cycle and can propagate by autoinfection, is excreted in large numbers with faeces, and has a low infectious dose; these factors attribute for its spread and ubiquity in the environment $[6,7]$. Transmission of Cryptosporidium is mainly through the faecal-oral route, as well as through contaminated water and food, person-to-person spread and contact with infected animals [8]. This parasite infection in immunodeficient patients may result in severe and chronic diarrhea, dehydration, wasting and death $[9,10]$. As no effective therapy is currently available, the need to prioritize interventions that decrease the risk of acquiring Cryptosporidium infection have to be emphasized. In this regard, early initiation of antiretroviral therapy (ART) in HIV infected patients helps restore immunity and prevents acquisition and/or facilitates clearance of an established infection [11].

In Ethiopia, most health facilities that provide HIV care and treatment services routinely screen all HIVinfected persons for non-opportunistic intestinal parasites at enrolment in the ART clinic as well as those with clinical indications during follow-up. However, opportunistic intestinal parasites are poorly addressed and no routine screening service is provided. We previously reported the high prevalence of protozoal and helminthic infections among HIV-infected people in southern Ethiopia where the infection rates for Cryptosporidium and S. stercoralis were shown to be up to 25 and $12.6 \%$, respectively [5, 12].

Improved care and treatment provided to people living with HIV/AIDS (PLHIV) has greatly decreased HIVrelated morbidities and mortalities in developing countries. Currently, in Ethiopia, HIV-infected persons initiate ART at CD4 T cell count $<500$ cells/ $\mu$ l compared to $<200$ cells $/ \mu \mathrm{l}$ and $<350$ cells $/ \mu \mathrm{l}$ in the year before 2009 and 2013, respectively. Moreover, intervention measures have been undertaken in order to reduce the risk of infection with intestinal parasites and decrease their clinical consequences in HIV-infected people. Since the year 2013, PLHIV have been provided with household water treatment (sodium hypochlorite solution with the brand name Wuha Agar and a flocculent-disinfectant product with the brand name $\mathrm{PuR}$ ), safe water storage using a narrowmouth container with tap, soap, anti-helminthic drugs and oral rehydration salt free of charge. Whether such interventions have reduced the public health significance of opportunistic or other intestinal parasites has yet to be systematically evaluated. This study was, therefore, conducted to determine the prevalence of Cryptosporidium and other intestinal parasitic infections among HIVinfected patients who receive care at one of the largest hospitals in southern Ethiopia where previous available baseline data helps evaluate if improved HIV-related care has reduced the rate of infection.

\section{Methods}

A cross-sectional study was conducted at Hawassa University Hospital from May, 2013 to March, 2014. The hospital is situated in Hawassa, the Capital City of the Southern Nations, Nationalities and Peoples' Regional State in Ethiopia. It is the largest hospital in the administrative region and provides care and treatment to $6230 \mathrm{HIV}$-infected people. Clinical and laboratory investigations including immunological (CD4 $\mathrm{T}$ cell count) and/or biochemical assessments at enrolment and on three-monthly follow-up visits help determine patient's eligibility for ART as well as monitor response to treatment.

The study population consisted of consecutive HIVinfected individuals who monitor their disease status at the ART clinic of the Hospital and with a CD4 T cell count $<200$ cells/ $\mu \mathrm{l}$ and/or with a complaint of diarrhea. A total of 491 individuals that fulfilled the specified inclusion criteria were prospectively recruited. Persons who took anti-parasitic treatment within a month prior to the time of data collection were excluded.

Counselor nurses interviewed the study participants collected socio-demography, risk factor, current symptom and duration of diarrhea data using structured questionnaires. Diarrhea was defined as watery or loose stool passed at least three times over a $24-\mathrm{h}$ period. The persistence of diarrhea for more than 14 days was defined as chronic; while, diarrhea that lasts less than 14 days was acute. All participants provided a single stool sample on the same day of interview and measurement of CD4 T cells. The stool samples were processed using direct microscopy (normal saline and iodine preparations) and formol-ether concentration techniques to detect trophozoite/cyst, larvae and ova of intestinal parasites as appropriate. The modified Ziehl-Neelsen staining technique was performed to detect oocysts of intestinal coccidian parasites, as described elsewhere [13]. In brief, air-dried stool smears were fixed with methanol for $3 \mathrm{~min}$, stained by carbol fuchsine for $15 \mathrm{~min}$, decolorized with $1 \%$ acid alcohol for $15 \mathrm{~s}$, and counterstained with $0.5 \%$ methylene blue for $30 \mathrm{~s}$. Stained smears were air-dried and examined microscopically (using 100x objective) for oocysts.

The current data were compared with previous data collected from November, 2008 to March, 2009. During 
this period, HIV-infected patients had been initiating ART at a CD4 level $<200$ cells/ $\mu$ l. In both studies, single stool samples were collected from the study participants and analyzed using the same laboratory techniques. HIV-infected individuals enrolled in the previous study were naïve for ART and regardless of diarrheal status or level of CD4 $\mathrm{T}$ cell count. In comparison, the present study investigated only patients with a CD4 T cell count $<200 \mathrm{cells} / \mu \mathrm{l}$ and/or with a complaint of diarrhea. It was shown in the previous study that these subgroups were more affected by opportunistic or any intestinal parasitic infections [5].

Data entry and analysis was performed using SPSS Version-16. Descriptive summaries were presented in terms of mean, range and proportions as appropriate. Differences between proportions were evaluated using Pearson's Chi-square test $\left(\chi^{2}\right)$, and a $P$-value $<0.05$ was considered significant.

The study was approved by the Institutional Review Board of the College of Medicine and Health Sciences, Hawassa University. Participation was voluntary and informed written consent was obtained from each study participant. Participants were informed of the confidentiality of their personal data, and physicians managed those found to be infected with any pathogenic intestinal parasite.

\section{Results}

A total of 491 HIV-infected individuals were enrolled during the study period. Most study participants were urban residents $(92.9 \%)$ and females $(60.7 \%)$. The mean age of study participants was 33.1 years (range, 15-70; SD, 9.51). Concerning ART status, $55.8 \%$ of the participants were on ART and the remaining $44.2 \%$ were ART-naive (Table 2). Individuals with a level of CD4 T cell count $<200$ cells $/ \mu$ l accounted for $56.2 \%$ of the participants. The remaining participants $(43.8 \%)$ were those who reported some type of diarrhea (acute or chronic). Of the participants with a CD4 level $<200$ cells $/ \mu$ l, the majority (65.2\%) reported no diarrhea, while 18.1 and $16.7 \%$ experienced acute and chronic diarrhea, respectively (Table 3).

The cumulative prevalence of intestinal parasitic infections in the study population was $35.8 \%$ (176/491). Individuals infected with protozoans accounted for $28.1 \%$ of the study participants while $11 \%$ were infected with helminths. The most prevalent parasites were Cryptosporidium (13.2 \%), followed by Entamoeba histolytica/dispar $(10.2 \%)$ and Giardia lamblia $(7.9 \%)$. The same rate of infection (4.5\%) was observed for Ascaris lumbricoides and Strongyloides stercoralis while the rate for Isospora belli was $2.2 \%$. The species-wise prevalence of different intestinal parasites in the study population is presented in Fig. 1. The rates of single and multiple parasitic infections in the study population were $25.5 \%$ and 10.4\%, respectively (Fig. 2).

The rate of intestinal parasitic infection was higher among rural residents (45.7\%), participants in the age range $40-49$ years $(44.3 \%)$, males (36.3\%), and in those who had no work (41.8\%). Nonetheless, only educational level of the participants was found to be significantly associated with rate of intestinal parasitic infection $\left(\chi^{2}=18.03, \mathrm{x} d f=3, P<0.001\right)$ where illiterates were found to be more affected (50.6\%) (Table 1).

The majority of the study participants used pipe water for both domestic and laundry purposes $(95.5 \%)$ and reported no contact with animals $(74.3 \%)$. The rate of Cryptosporidium infection among participants in the age group 15-19 years, ART-naïve, had animal contact, and used pipe water were $15.8,15.2,15.1$ and $13.6 \%$, respectively. However, none of these factors was found to be associated with Cryptosporidium infection (Table 2).

The distribution of any intestinal parasitic infection or cryptosporidiosis in relation to diarrheal status and CD4 $\mathrm{T}$ cell count is presented in Table 3 . The majority $(65.2 \%)$ of the study participants with a CD4 T cell count lower than 200 cells/ $\mu$ l reported no diarrhea. Participants with the lower CD4 $\mathrm{T}$ cell count had a similar rate of any intestinal parasitic infection (36.2 \%) and cryptosporidiosis (15.6\%) compared to those with a CD4 count $\geq 200$ cells/ $\mu$ l, but with some type of diarrhea (parasitic infection, $35.6 \%$; cryptosporidiosis, $10.2 \%$ ). In participants categorized by a CD4 T cell count, the rate of parasitic infection was higher in patients with chronic diarrhea. However, a statistically significant difference in rate of any parasitic infection by type of diarrhea was observed in a CD4 category 200-499 cells/ $\mu \mathrm{l}$ (acute diarrhea $26.2 \%$ vs chronic diarrhea $45.2 \% ; \chi^{2}=4.99, d f=1$, $P=0.03)$. Similarly, difference in rate of Cryptosporidium infection by diarrhea status was statistically significant in patients with a CD4 level $<200$ cells $/ \mu$ l (no diarrhea $15 \%$, acute diarrhea $6 \%$, chronic diarrhea $28.3 \%$; $\chi^{2}=$ 9.12, $d f=2, P=0.01$ ).

\section{Discussion}

In this cross-sectional study, the cumulative prevalence of intestinal parasitic infection in the study population was found to be $35.8 \%$, which is comparable with a result reported in eastern Ethiopia (33.7 \%) [14]. However, the current finding is significantly lower compared to a previous study in the same hospital (59.8\%) [5] or another study in Bahirdar (northwest Ethiopia) (69 \%) [15]. The lower rate of infection in the current report is likely to be the outcome of improved care and treatment provided to people living with HIV/AIDS. In light of the relatively high risk population investigated in this study, the result is encouraging and emphasizes the importance of sustaining such intervention efforts. 


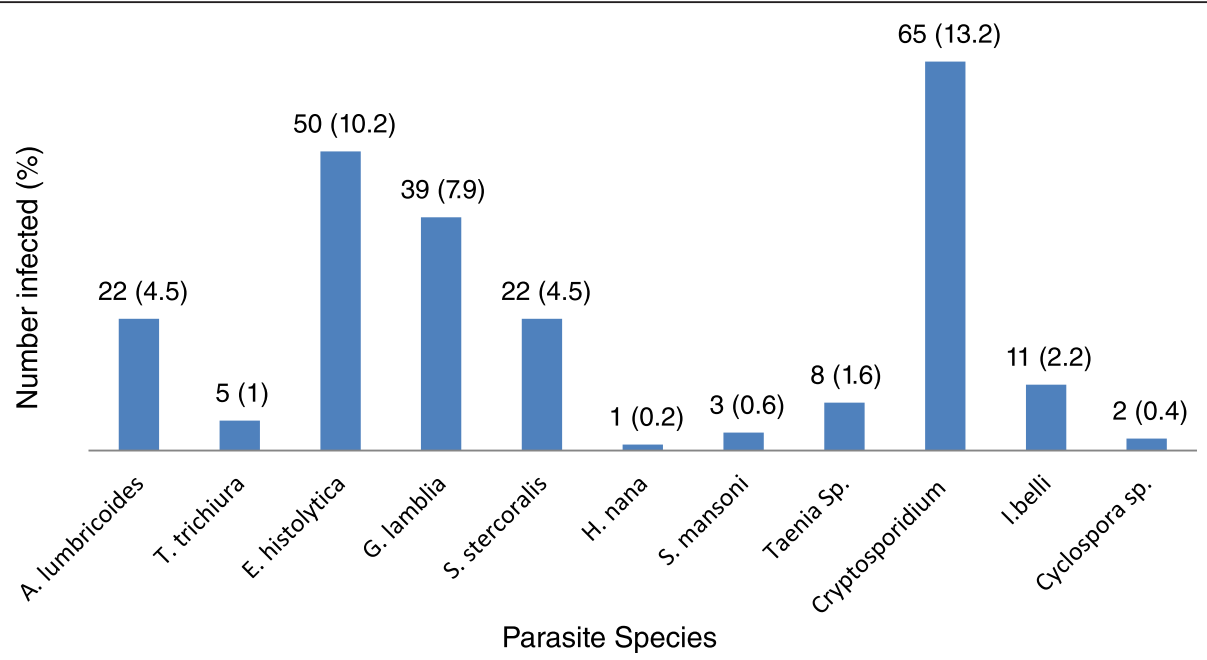

Fig. 1 Prevalence of different species of intestinal parasites in the study population in Hawassa University Hospital, southern Ethiopia, 2013-2014

As to the diversity of parasite species, several reports in Ethiopia showed similar results where helminths like A. lumbricoides and S. stercoralis and protozoan parasites such as Cryptosporidium, E. histolytica/dispar and G. lamblia were dominating species in the study population $[5,12,14,15]$. However, compared to our previous study in the same hospital [5], the current prevalence of the specified parasites has decreased considerably: $A$. lumbricoides (12.2 vs $4.5 \%$ ) and S. stercoralis (12.6 vs $4.5 \%)$. The rate of $S$. stercoralis infection in the current study was also lower than previous findings in Ethiopia: $12 \%$ in Yirgalem (southern Ethiopia) [12], $10.7 \%$ in Gondar (northern Ethiopia) [16], and $7.8 \%$ in Jimma (southwestern Ethiopia) [17]. Similarly, protozoan parasites were detected at lower rates in the current study compared to the previous study conducted in the same hospital: $E$. histolytica/dispar (previous 24.8 vs current $10.2 \%$ ) and G. lamblia (11.2 vs $7.9 \%$ ). The decreased rate of cryptosporidiosis (50.1 vs $15.6 \%$ ) and isosporiasis (12.2 vs $2.2 \%)$ among patients with a CD4 T cell count $<200$ cells/ $\mu l$ may indicate the success of intervention efforts in reducing the significance of opportunistic intestinal parasitic infections in the specified high risk group. However, in contrast to our current report, the rate of cryptosporidiosis (26.9\%) among HIV-infected patients in Addis Ababa (central Ethiopia) was higher, which may partly be due to the sensitive laboratory technique (PCR-RFLP analysis) employed in the study [18]. Overall,

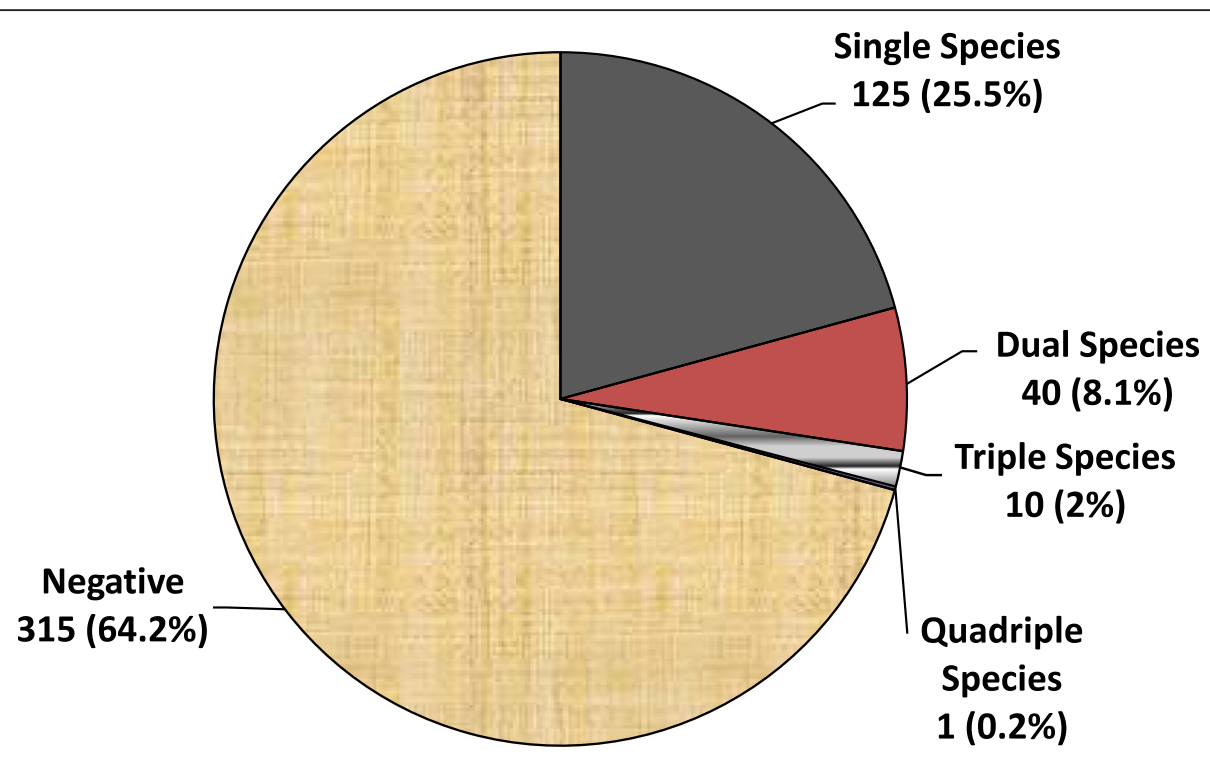

Fig. 2 Prevalence of single and multiple parasite infections in the study population in Hawassa University Hospital, southern Ethiopia, 2013-2014 
Table 1 Intestinal parasitic infection in relation to sociodemography in the study population in Hawassa University Hospital, southern Ethiopia, 2013-2014

\begin{tabular}{|c|c|c|c|c|}
\hline Characteristics & $\begin{array}{l}\text { Number (\%) } \\
\text { tested }\end{array}$ & $\begin{array}{l}\text { Number (\%) } \\
\text { positive for } \\
\text { any parasite }\end{array}$ & $x^{2}$ & $P$-value \\
\hline \multicolumn{5}{|l|}{ Residence } \\
\hline Rural & $35(7.1)$ & $16(45.7)$ & 1.53 & 0.22 \\
\hline Urban & $456(92.9)$ & $161(35.3)$ & & \\
\hline \multicolumn{5}{|l|}{ Age group (years) } \\
\hline $15-19$ & $19(3.9)$ & $7(36.8)$ & 5.32 & 0.26 \\
\hline $20-29$ & $165(33.6)$ & $63(38.2)$ & & \\
\hline $30-39$ & $186(37.9)$ & $58(31.2)$ & & \\
\hline $40-49$ & $88(17.9)$ & $39(44.3)$ & & \\
\hline$\geq 50$ & $33(6.7)$ & $10(30.3)$ & & \\
\hline \multicolumn{5}{|l|}{ Sex } \\
\hline Male & $193(39.3)$ & $70(36.3)$ & 0.07 & 0.94 \\
\hline Female & $298(60.7)$ & $107(35.9)$ & & \\
\hline \multicolumn{5}{|l|}{ Occupation } \\
\hline $\begin{array}{l}\text { Government } \\
\text { employee }\end{array}$ & $73(14.9)$ & $23(31.5)$ & 6.55 & 0.623 \\
\hline $\begin{array}{l}\text { Non-governmental } \\
\text { employee }\end{array}$ & $47(9.6)$ & $11(23.4)$ & & \\
\hline House wife & $133(27.1)$ & $55(41.4)$ & & \\
\hline Student & $13(2.6)$ & $5(38.5)$ & & \\
\hline Merchant & $85(17.3)$ & $31(36.5)$ & & \\
\hline Housemaid & $28(5.7)$ & $10(35.7)$ & & \\
\hline No work & $55(11.2)$ & $23(41.8)$ & & \\
\hline Other & $57(11.6)$ & $19(33.3)$ & & \\
\hline \multicolumn{5}{|l|}{ Educational status } \\
\hline Illiterate & $89(18.1)$ & $45(50.6)$ & 18.01 & $<0.0001$ \\
\hline Primary & $143(29.1)$ & $60(42)$ & & \\
\hline Secondary & $198(40.3)$ & $56(28.3)$ & & \\
\hline Tertiary & $61(12.4)$ & $16(26.2)$ & & \\
\hline
\end{tabular}

the decreased rate of infection for different parasites may reflect the impact of the current intervention measures on various type of parasites with clinical and public health significance in HIV-infected people. It was possibly due to the decreased rate of occurrence of various parasitic species that the rate of multiple infections $(10.4 \%)$ in our current study was lower compared to that reported in our previous one (27.2\%) [5] or another study elsewhere (93.1\%) [15]. This report, therefore, encourages public health officials and other concerned bodies to further strengthen the existing HIV-related care and treatment program so that parasite-associated morbidity and mortality can be diminished.
Table 2 Distribution of Cryptosporidium infection in relation to risk factors in the study population in Hawassa University Hospital, southern Ethiopia, 2013-2014

\begin{tabular}{lcccc}
\hline Characteristics & $\begin{array}{l}\text { Number } \\
(\%) \text { tested }\end{array}$ & $\begin{array}{c}\text { Number (\%) positive } \\
\text { for Cryptosporidium }\end{array}$ & $x^{2}$ & P-value \\
\hline Water source & & & & \\
Pipe & $471(95.9)$ & $64(13.6)$ & 1.31 & 0.52 \\
Well & $17(3.5)$ & $1(5.9)$ & & \\
Lake & $3(0.6)$ & $0(0.0)$ & & \\
Animal contact & & & & \\
Yes & $126(25.7)$ & $19(15.1)$ & 0.5 & 0.48 \\
No & $365(74.3)$ & $46(12.6)$ & & \\
ART status & & & & \\
ART naïve & $217(44.2)$ & $33(15.2)$ & 1.31 & 0.25 \\
On ART & $274(55.8)$ & $32(11.7)$ & & \\
Age group (years) & & & & \\
$15-19$ & $19(3.9)$ & $3(15.8)$ & 0.84 & 0.93 \\
$20-29$ & $165(33.6)$ & $19(11.5)$ & & \\
$30-39$ & $186(37.8)$ & $26(14.0)$ & & \\
$40-49$ & $88(17.9)$ & $13(14.8)$ & & \\
$\geq 50$ & $33(6.7)$ & $4(12.1)$ & & \\
\hline
\end{tabular}

Gastroenteritis is one of the major HIV-related illnesses and intestinal parasites are the most important agents implicated. We previously reported the significance of either HIV or parasitic infections independently or as coinfections to cause any type of diarrhea or chronic diarrhea [5]. It is routine clinical practice to screen patients with diarrhea for intestinal parasitic infections, although opportunistic parasites are usually overlooked in our context where laboratory services are limited. A similar rate of either any parasitic infection or cryptosporidiosis in patients with a CD4 $\mathrm{T}$ cell count $<200$ cells $/ \mu$ l compared to those with a CD4 count $\geq 200$ cells/ $\mu$ l, but with some type of diarrhea highlights the importance of providing a screening service for all patients with a CD4 count $<200$ cells/ $\mu \mathrm{l}$ regardless of their diarrheal status. A higher rate of parasitic infection was observed among patients that had chronic diarrhea, although the difference was found to be statistically significant only in the CD4 T cell count category 200-499 cells/ $\mu$ l. Similarly, the association of Cryptosporidium infection with diarrheal status was found to be statistically significant in patients with a CD4 level $<200$ cells $/ \mu \mathrm{l}$ in which the parasite was more likely to be detected in those who had chronic diarrhea. These results indicate that the chance of detecting intestinal parasites is higher among patients with chronic diarrhea. The detection rate of Cryptosporidium parasite was also significantly higher among patients with lower CD4 level, but with chronic diarrhea. 
Table 3 Distribution of any parasite and Cryptosporidium infections in relation to diarrheal status and CD4 T-cell count in the study population in Hawassa University Hospital, southern Ethiopia, 2013-2014

\begin{tabular}{|c|c|c|c|c|c|c|}
\hline CD4+ count $/ \mu \mathrm{l}$ & Diarrhea status & No. tested (\%) & No. positive for any parasite (\%) & $P$-value & No. positive for Cryptosporidium & $P$-value \\
\hline \multirow[t]{4}{*}{$<200$} & No & 180(65.2) & $63(35)$ & 0.16 & $27(15)$ & 0.01 \\
\hline & Acute & $50(18.1)$ & $15(30)$ & & $3(6)$ & \\
\hline & Chronic & $46(16.7)$ & $22(47.8)$ & & 13(28.3) & \\
\hline & Total & $276(56.2)$ & $100(36.2)$ & & $43(15.6)$ & \\
\hline \multirow[t]{2}{*}{$200-499$} & Acute & 103(71) & $27(26.2)$ & 0.03 & $7(6.8)$ & - \\
\hline & Chronic & $42(29)$ & 19(45.2) & & $9(1.4)$ & \\
\hline \multirow[t]{3}{*}{$\geq 500$} & Acute & $56(80)$ & 24(42.9) & 0.63 & $5(8.9)$ & - \\
\hline & Chronic & $14(20)$ & $7(50)$ & & $1(7.1)$ & \\
\hline & Total & $215(43.8)$ & $77(35.8)$ & & $22(10.2)$ & \\
\hline
\end{tabular}

Various factors such as animal-human mixing patterns, access to safe drinking water, immunity and ART status of the study population have been reported to influence the epidemiology of cryptosporidiosis [7, 8, 11]. In agreement, reports in Ethiopia showed a lower rate of cryptosporidiosis among HIV-infected patients on ART compared to those who were naïve for ART [14, 19]. As the aim of the current investigation was to assess the status of intestinal parasitic infections in the context of improved HIV-related care, no attempt was made to establish matching subgroups for making valid comparisons. It is, therefore, difficult to reason why the rate of cryptosporidiosis was not found to be associated with factors including ART status, contact with animals and source of water in the current investigation. Which specific interventions or factors impacted the prevalence of parasitic infections needs to be investigated.

This study had some limitations in light of which its results need to be interpreted. First, as a single stool specimen was examined, the study did not benefit from examining multiple samples, which could improve the rate of detection. Secondly, the modified Ziehl-Neelsen staining technique has sensitivity inferior to methods such as polymerase chain reaction and direct fluorescent-antibody tests; thus, prevalence of cryptosporidiosis may be underestimated. Thirdly, incapability to further characterize isolates of Cryptosporidium deprives the possibility of defining the source of infection as well as routes of transmission in the study population. Lastly, the role of other diarrhea causing bacterial and viral pathogens was not addressed.

\section{Conclusion}

The study shows a high prevalence of intestinal parasitic infections in the study population. However, the results are significantly lower compared to previous findings in the same hospital. The observed decreased rate of infection is most likely to be the outcome of intervention measures undertaken as part of improved HIV-related care in our health institution. Thus, it encourages the need to strengthen and sustain the existing intervention measures so as to further reduce the significance of intestinal parasitic infections in people living with HIV/ AIDS. The current study also emphasizes the importance of screening HIV-infected patients for Cryptosporidium infection; with the priority given to patients with diarrhea as well as to those with a CD4 count $<200$ cells/ $\mu$ l regardless of their diarrheal status.

\section{Competing interests}

The authors declare that they have no competing interests.

\section{Authors' contributions}

TS and TL designed the study and contributed to the laboratory work; TS and YT performed the statistical analyses, interpretation and contributed to the write up. All authors read and approved the final version of the manuscript.

\section{Acknowledgments}

The authors would like to acknowledge the International Society for Infectious Diseases and the Hawassa University for financial support. We extend our appreciation to the counselor nurses and laboratory personnel, especially Mr. Demissie Assegu for assistance during data collection, and to the study participants who voluntarily took part in the study. Finally, we are most thankful to Dr. Justine Pulford, who kindly read and edited this manuscript.

Received: 20 November 2015 Accepted: 28 April 2016

Published online: 10 May 2016

\section{References}

1. Framm SR, Soave R. Agents of diarrhea. Med Clin North Am. 1997;81:427-47.

2. Cimerman S, Cimerman B, Lewi SD. Enteric parasites and AIDS. Sao Paulo Med J. 1999;117:266-73.

3. Arora DR, Arora B. AIDS - associated parasitic diarrhoea. Indian J Med Microbiol. 2009;27:185-90.

4. Dufera M, Petros B, Endeshaw T, Mohammed H, Kassu A. Opportunistic intestinal protozoan parasites among HIV positive patients on antiretroviral therapy at Nekemte Hospital, West Ethiopia. Ethiop J Health Biomed Sci. 2008;1:11-7

5. Assefa S, Erko B, Medhin G, Assefa Z, Shimelis T. Intestinal parasitic infections in relation to HIV/AIDS status, diarrhea and CD4 T-cell count. BMC Infect Dis. 2009;9:155.

6. Snelling W, Xiao L, Ortega-Pierres G, Lowery JC, Moore JE, Rao JR, Smyth S, Millar CB, Rooney PJ, Matsuda M, Kenny F, Xu J, Dooley JSG. Cryptosporidiosis in developing countries. J Infect Developing Countries. 2007;1:242-56. 
7. Xiao L. Molecular epidemiology of cryptosporidiosis: An update. Exp Parasitol. 2010;124:80-9.

8. Fayer R, Morgan U, Upton JS. Epidemiology of Cryptosporidium: transmission, detection and identification. Int J Parasitol. 2000;30:1305-22.

9. Dionisio D. Cryptosporidiosis in HIV-infected patients. J Postgrad Med. 2002; 48:215-8.

10. Hunter PR, Nichols G. Epidemiology and clinical features of Cryptosporidium infection in immunocompromised patients. Clin Microbiol Rev. 2002;15:145-54.

11. Zardi EM, Picardi A, Afeltra A. Treatment of cryptosporidiosis in immunocompromised hosts. Chemotherapy. 2005;51:193-6.

12. Getaneh A, Medhin G, Shimelis T. Cryptosporidium and Strongyloides stercoralis infections among people with and without HIV infection and efficiency of diagnostic methods for Strongyloides in Yirgalem Hospital, southern Ethiopia. BMC Res Notes. 2010;3:90.

13. Shimelis T, Tadesse E. Performance evaluation of point-of-care test for detection of Cryptosporidium stool antigen in children and HIV infected adults. Parasit Vectors. 2014;7:227.

14. Teklemariam Z, Abate D, Mitiku H, Dessie Y. Prevalence of intestinal parasitic infection among HIV positive persons who are naive and on antiretroviral treatment in Hiwot Fana Specialized University Hospital, Eastern Ethiopia. ISRN AIDS. 2013;11(2013):324329.

15. Moges F, Kebede Y, Kassu A, Degu G, Tiruneh M, Gedefaw M. Infection with HIV and intestinal parasites among street dwellers in Gondar city, northwest Ethiopia. Jpn J Infect Dis. 2006;59:400-3.

16. Alemu A, Shiferaw Y, Getnet G, Yalew A, Addis Z. Opportunistic and other intestinal parasites among HIV/AIDS patients attending Gambi Higher Clinic in Bahirdar City, North West Ethiopia. Asian Pac J Trop Med. 2011;4:661-5.

17. Awole M, Gebre-Selassie S, Kassa T, Kibru G. Prevalence of intestinal parasites in HIV- infected adult patients in South Western Ethiopia. Ethiop J Health Dev. 2003;17:71-8.

18. Adamu H, Petros B, Zhang G, Kassa H, Amer S, Ye J, Feng Y, Xiao L. Distribution and clinical manifestations of Cryptosporidium species and subtypes in HIV/ AIDS patients in Ethiopia. PLoS Negl Trop Dis. 2014;17(8):e2831.

19. Missaye A, Dagnew M, Alemu A, Alemu A. Prevalence of intestinal parasites and associated risk factors among HIV/AIDS patients with pre-ART and onART attending Dessie Hospital ART Clinic, Northeast Ethiopia. AIDS Res Ther. 2013;25(10):7.

\section{Submit your next manuscript to BioMed Central and we will help you at every step:}

- We accept pre-submission inquiries

- Our selector tool helps you to find the most relevant journal

- We provide round the clock customer support

- Convenient online submission

- Thorough peer review

- Inclusion in PubMed and all major indexing services

- Maximum visibility for your research

Submit your manuscript at www biomedcentral.com/submit

) Biomed Central 